\title{
Cognitive Status Correlates with CXCL10/IP-10 Levels in Parkinson's Disease
}

\author{
Natália Pessoa Rocha, ${ }^{1,2}$ Paula Luciana Scalzo, ${ }^{3}$ Izabela Guimarães Barbosa, ${ }^{1}$ \\ Mariana Soares Souza, ${ }^{4}$ Isabela Boechat Morato, ${ }^{1}$ Érica Leandro Marciano Vieira, ${ }^{1}$ \\ Paulo Pereira Christo, ${ }^{4}$ Antônio Lúcio Teixeira, ${ }^{1}$ and Helton José Reis ${ }^{2}$ \\ ${ }^{1}$ Laboratório Interdisciplinar de Investigação Médica (LIIM), Faculdade de Medicina, Universidade Federal de Minas Gerais, \\ Avenida Prof. Alfredo Balena 190, Sala 281, 30130-100 Belo Horizonte, MG, Brazil \\ ${ }^{2}$ Laboratório de Neurofarmacologia, Instituto de Ciências Biológicas, Universidade Federal de Minas Gerais, \\ Avenida Presidente Antônio Carlos 6627, 31270-901 Belo Horizonte, MG, Brazil \\ ${ }^{3}$ Laboratório de Neurobiologia, Instituto de Ciências Biológicas, Universidade Federal de Minas Gerais, Avenida Presidente Antônio \\ Carlos 6627, 31270-901 Belo Horizonte, MG, Brazil \\ ${ }^{4}$ Departamento de Neurologia e Neurocirurgia, Santa Casa de Belo Horizonte Hospital, Avenida Francisco Sales 1111, \\ 30150-221 Belo Horizonte, MG, Brazil
}

Correspondence should be addressed to Natália Pessoa Rocha; npessoarocha@gmail.com

Received 19 August 2014; Revised 19 September 2014; Accepted 20 September 2014; Published 15 October 2014

Academic Editor: Heinz Reichmann

Copyright (C) 2014 Natália Pessoa Rocha et al. This is an open access article distributed under the Creative Commons Attribution License, which permits unrestricted use, distribution, and reproduction in any medium, provided the original work is properly cited.

Cognitive impairment and depressive symptoms are of great interest in Parkinson's disease (PD), since they are very common and lead to increased disability with poor quality of life. Inflammatory mechanisms have been implicated in PD and its nonmotor symptoms. In the current pilot study, we aimed to evaluate plasma levels of chemokines in PD patients and to analyze the putative association of chemokines with depressive symptoms and cognitive performance. We hypothesized that higher chemokines levels are associated with worse cognitive performance and increased depressive symptoms in PD. For this purpose, 40 PD patients and 25 age- and gender-matched controls were subjected to a clinical evaluation including cognitive and mood tests. Peripheral blood was drawn and plasma levels of CCL2/MCP-1, CCL11/eotaxin, CCL24/eotaxin-2, and CXCL10/IP-10 were measured by enzymelinked immunosorbent assay. PD patients and control individuals presented comparable plasma concentrations of all the evaluated chemokines. In PD patients, CXCL10/IP-10 plasma levels correlated positively with Hoehn and Yahr staging scale. In addition, the higher CXCL10/IP-10 levels, the worse performance on cognitive tests. Although there was no significant difference between PD patients and control individuals regarding chemokines levels, our preliminary results showed that CXCL10/IP-10 may be associated with cognitive status in PD.

\section{Introduction}

Parkinson's disease (PD) is the second most common neurodegenerative disorder worldwide. PD is characterized by the progressive loss of dopaminergic neurons of the substantia nigra pars compacta (SNpc) and the presence of alphasynuclein intraneuronal inclusions called Lewy bodies in the remaining neurons [1]. It is well known that genetic mutations can cause familial parkinsonism, but only $10 \%$ of PD cases have a clear genetic origin. The etiopathogenesis of PD remains inconclusive in the great majority of cases [2]. Among several proposed causes of neuronal death in PD, mitochondrial dysfunction, oxidative stress, environmental toxins, and immune/inflammatory responses may be relevant candidates. The contribution of neuroinflammation (i.e., microglia activation, leukocytes infiltration, and increased levels of inflammatory mediators) to the pathophysiology of PD was first described in postmortem studies [3, 4]. Epidemiological, genetic, and immunological studies in humans and animal models have shown that not only neuroinflammation 
but also peripheral inflammatory changes may contribute to PD onset and development [5]. Lately, it has been shown that peripheral inflammatory and immune changes described in PD may be associated with some of the clinical signs, especially nonmotor symptoms, experienced by PD patients [6-9].

Chemokines are interesting molecular candidates to be studied in PD. Chemokines are chemotactic cytokines; that is, they attract and activate immune and nonimmune cells. For instance, they act as immune mediators, regulating leukocyte infiltration into the brain during inflammatory or infectious diseases [10]. A range of studies has also suggested that besides the well-established role in the immune system, chemokines and their receptors may also play an important role in the central nervous system (CNS). Chemokines and their receptors may modulate neurotransmitter release, regulating synaptic transmission, and cell survival. Guyon and colleagues demonstrated that the injection of CCL2/MCP-1 (monocyte chemotactic protein 1) onto dopaminergic neurons in the SNpc of rats increased cell excitability, dopamine release, and related locomotor activity [11]. Therefore, chemokines may represent a new class of neuromodulators, especially in dopaminergic neurons, potentially representing new targets for the treatment of PD. In addition, one post mortem study found that, despite the loss of dopaminergic neurons, the SNpc of PD patients exhibited increased levels of CXCR4 and its ligand CXCL12/SDF-1 (stromal cellderived factor 1) in comparison with controls. Experiments in 1-methyl-4-phenyl-1,2,3,6-tetrahydropyridine- (MPTP-) induced PD mice confirmed these results, showing that MPTP produced a time-dependent upregulation of CXCR4 that preceded the loss of dopaminergic neurons [12]. Genetic studies also suggested the involvement of chemokines in PD. For instance, a single nucleotide polymorphism of the CXCL8/interleukin- (IL-) 8 A-251T gene was associated with $\mathrm{PD}$ in the Irish population [13]. Changes in the peripheral levels of chemokines such as CCL5/RANTES (acronym for regulated on activation, normal $\mathrm{T}$ cell expressed and secreted), CCL2/MCP-1, CCL3/MIP-1 $\alpha$ (macrophage inflammatory protein-1 $\alpha$ ), and CXCL8/IL- 8 have been demonstrated in PD in comparison with controls [14, 15]. The dysregulation of chemokines is described in several neuropsychiatric disorders such as major depression [16], bipolar disorder [17], schizophrenia [18], obsessive-compulsive disorder [19], and Alzheimer's disease (AD) [20].

Although PD is largely characterized by specific motor signs, nonmotor symptoms are also frequent and disabling. Among these, cognitive impairment and depressive states are of great interest since they are common, progress as the disease advances, and lead to increased disability with poor quality of life [21-23]. Chemokines may influence functions such as mood, behavior, and cognition, possibly related to their neuromodulatory and/or direct neurotransmitter-like effects, regulation of neuroendocrine axes, control of the blood-brain barrier (BBB) permeability, and regulation of neurogenesis (for a review, see [24]). In the current study, we aimed to evaluate the plasma levels of chemokines in PD patients and to investigate the putative association of their levels with depressive symptoms and performance in cognitive tests. We hypothesized that higher chemokines levels are associated with worse cognitive performance and more depressive symptoms in PD.

\section{Material and Methods}

2.1. Subjects. This study included $40 \mathrm{PD}$ patients diagnosed according to the United Kingdom PD Brain Bank criteria [25] and a group of 25 healthy controls matched by age, gender, body mass index (BMI), and educational level. Patients were recruited from the Outpatient Movement Disorders Clinic of the "Santa Casa de Belo Horizonte" Hospital, Belo Horizonte, Brazil. Controls were recruited from the local community. Participants were excluded if they had undergone previous neurosurgery or if they had any other neurological disorder and/or cognitive decline (i.e., delirium or dementia), significant sensory impairment, and infectious or autoimmune diseases in activity in the previous four weeks. In addition, individuals who had used corticosteroids, antiinflammatories, or antibiotics in the four weeks prior to the study were excluded. All subjects provided written informed consent before admission to the study. The Research Ethics Committee of the Universidade Federal de Minas Gerais, Brazil, approved this study.

2.2. Clinical Evaluation. All patients were evaluated with the Unified Parkinson's Disease Rating Scale (UPDRS) [26] which assesses different signs and symptoms of PD. The UPDRS scores were obtained in the "on" state of the disease. The modified Hoehn and Yahr staging scale (HY) was used to establish the stage of PD [27]. The modified Schwab and England activities of daily living (ADL) scale ( $\& \& E$ ) were used to assess daily routine of PD patients [26].

All individuals were subjected to cognitive examination which included the Mini-Mental State Examination (MMSE) [28] adapted for the Brazilian elderly population. MMSE is a brief test for cognitive screening, comprising items from different domains such as orientation, attention, memory, and language. Since impairment in executive functioning is the most common cognitive deficit in PD patients, the Frontal Assessment Battery (FAB) was also used [29]. FAB is a brief assessment tool that evaluates executive functioning and consists of six subtests exploring cognitive processes related to the frontal lobes: conceptualization mental flexibility, motor programming, sensitivity to interference, inhibitory control, and environmental autonomy. In each subtest, scores range from 0 (worst) to 3 (best). The total FAB score is calculated by the sum of the scores of each of the six subtests. In addition, all participants were evaluated using Beck's Depression Inventory (BDI), a self-rating instrument for depressive symptoms comprising 21 items, each one ranging from 0 to 3 , according to the severity of symptoms [30]. BDI has been validated as a tool for depression screening and diagnosis in PD.

2.3. Chemokines Assessment. Ten milliliters of blood were drawn by venipuncture in vacuum tubes containing heparin (Vacuplast, Huangyn, China) on the same day of the clinical 
TABLE 1: Demographic and nonmotor features of Parkinson's disease (PD) patients and control subjects.

\begin{tabular}{|c|c|c|c|}
\hline & PD patients $(n=40)$ & Control subjects $(n=25)$ & $P$ value \\
\hline Gender (female/male) & $13 / 27$ & $6 / 19$ & $0.58^{\mathrm{a}}$ \\
\hline Age in years (mean $\pm S D)$ & $68.71 \pm 10.07$ & $65.23 \pm 8.75$ & $0.20^{\mathrm{b}}$ \\
\hline Body mass index in $\mathrm{Kg} / \mathrm{m}^{2}($ mean $\pm \mathrm{SD})$ & $26.02 \pm 3.73$ & $27.64 \pm 3.71$ & $0.09^{c}$ \\
\hline Educational level in years (mean \pm SD) & $4.72 \pm 2.87$ & $6.72 \pm 5.37$ & $0.16^{\mathrm{b}}$ \\
\hline MMSE $[$ mean \pm SD (median)] & $24.00 \pm 3.99(25)$ & $27.00 \pm 3.57(29)$ & $0.001^{\mathrm{b}}$ \\
\hline $\mathrm{FAB}[$ mean $\pm \mathrm{SD}$ (median) $]$ & $11.49 \pm 2.99(12)$ & $12.32 \pm 3.67(13)$ & $0.32^{\mathrm{c}}$ \\
\hline Conceptualization & $1.23 \pm 1.01(1)$ & $1.64 \pm 1.11(2)$ & $0.12^{\mathrm{b}}$ \\
\hline Mental flexibility & $1.82 \pm 1.10(2)$ & $2.08 \pm 1.04(2)$ & $0.34^{\mathrm{b}}$ \\
\hline Programming & $1.74 \pm 0.91(2)$ & $2.24 \pm 0.83(2)$ & $0.04^{\mathrm{b}}$ \\
\hline Sensitivity to interference & $2.26 \pm 0.94(3)$ & $1.84 \pm 1.25(2)$ & $0.21^{\mathrm{b}}$ \\
\hline Inhibitory control & $1.41 \pm 0.88(1)$ & $1.52 \pm 1.09(1)$ & $0.73^{\mathrm{b}}$ \\
\hline Environmental autonomy & $3.00 \pm 0.00(3)$ & $3.00 \pm 0.00(3)$ & $1.00^{\mathrm{b}}$ \\
\hline $\mathrm{BDI}[$ mean $\pm \mathrm{SD}$ (median) $]$ & $8.64 \pm 7.58(6)$ & $2.76 \pm 3.35(1)$ & $<0.001^{\mathrm{b}}$ \\
\hline \multicolumn{4}{|l|}{ Medication in use (frequency in \%) } \\
\hline Antihypertensive (\%) & 55.00 & 48.00 & $0.62^{\mathrm{a}}$ \\
\hline Antidiabetic (\%) & 10.00 & 20.00 & $0.29^{\mathrm{a}}$ \\
\hline Hypolipidemic (\%) & 10.00 & 24.00 & $0.17^{\mathrm{a}}$ \\
\hline Levothyroxine (\%) & 10.00 & 4.00 & $0.64^{\mathrm{a}}$ \\
\hline Antidepressants & 20.00 & 12.00 & $0.51^{\mathrm{a}}$ \\
\hline
\end{tabular}

BDI: Beck Depression Inventory; FAB: Frontal Assessment Battery; MMSE: Mini-Mental State Examination; PD: Parkinson’s disease; SD: standard deviation. ${ }^{\mathrm{a}}$ Fisher's exact test; ${ }^{\mathrm{b}}$ Mann-Whitney test; ${ }^{\mathrm{c}}$ Student's $t$ test.

assessment. In order to rule out any confounding factors caused by circadian rhythm, all samples were collected at the same time of the day, between 14 and $16 \mathrm{~h}$. The whole blood samples were kept at room temperature and used within $2 \mathrm{~h}$ after having been drawn. These samples were then centrifuged at $3,000 \mathrm{~g}$ for $10 \mathrm{~min}, 4^{\circ} \mathrm{C}$, twice. The plasma was collected and stored at $-70^{\circ} \mathrm{C}$ until assayed. The samples were thawed and excess of proteins was removed by acid/salt precipitation as routinely performed in our laboratory $[17$, 31]. Briefly, we mixed equal volume of plasma and $1.2 \%$ trifluoroacetic acid $/ 1.35 \mathrm{~mol} / \mathrm{L} \mathrm{NaCl}$. The plasma/acid trifluoroacetic mixture was left at room temperature for 10 minutes. Afterward, the samples were centrifuged for 5 minutes at $10,000 \mathrm{rpm}$. We then adjusted the supernatants for salt content $(0.14 \mathrm{~mol} / \mathrm{L}$ sodium chloride and $0.01 \mathrm{~mol} / \mathrm{L}$ sodium phosphate) and $\mathrm{pH}$ (7.4), for the determination of chemokines concentration.

Plasma levels of CCL2/MCP-1, CCL11/eotaxin, CCL24/ eotaxin-2, and CXCL10/IP-10 (interferon gamma-induced protein 10) were measured by Enzyme-Linked Immunosorbent Assay (ELISA) according to the procedures supplied by the manufacturer (DuoSet, R\&D Systems, Minneapolis, MN, USA). Concentrations are expressed as pg/mL. Lower detection limits for all analyzed chemokines were $10 \mathrm{pg} / \mathrm{mL}$.

2.4. Statistical Analysis. Association between dichotomous variables was assessed with Fisher's exact test. All variables were tested for Gaussian distribution by the ShapiroWilk normality test. Two groups (patients versus controls) were compared by Mann-Whitney or Student's $t$ tests when nonnormally or normally distributed, respectively. Spearman's correlation analyses were performed to examine the relationship between clinical variables and plasma levels of chemokines. All statistical tests were two-tailed and were performed using a significance level of $\alpha=0.05$. Statistical analyses were performed using SPSS software version 16.0 (SPSS Inc., Chicago, IL, USA).

\section{Results}

3.1. Sociodemographic and Clinical Results. Demographic and nonmotor features of both groups are shown in Table 1. PD patients' clinical data are given in Table 2. PD patients presented a worse performance in the MMSE in comparison with controls $(Z=-3,325 ; P=0.001)$. There was no difference between $\mathrm{PD}$ and control individuals regarding total FAB performance. Nonetheless, the analysis of the subtests demonstrated that $\mathrm{PD}$ patients presented a lower score in program$\operatorname{ming}(Z=-2,107 ; P=0.04)$. In addition, BDI score was higher in PD patients compared to controls $(Z=-3,528$; $P<0.001$ ).

Regarding PD patients, there was a negative correlation between total FAB score and age $(\rho=-0.445, P=0.005)$. As expected, higher educational level was associated with better performance in both cognitive tests, MMSE and FAB ( $\rho=$ $0.429, P=0.006$, and $\rho=0.463, P=0.003$, resp.). BDI score correlated positively with UPDRS total score and HY stage ( $\rho=0.421, P=0.010$ and $\rho=0.440, P=0.006$, resp.) and negatively with S\&E scale $(\rho=-0.457, P=0.004)$. Considering control subjects, total FAB performance correlated negatively with age and BDI score $(\rho=-0.677, P<0.001$ and 
TABLE 2: Clinical features of Parkinson's disease (PD) patients.

\begin{tabular}{lc}
\hline & PD patients $(n=40)$ \\
\hline Length of illness in years [mean \pm SD (range)] & $5.45 \pm 4.13(0.4-18)$ \\
UPDRS [mean \pm SD (range)] & $51.82 \pm 25.27(11-105)$ \\
UPDRS I [mean \pm SD (range)] & $3.36 \pm 2.96(0-11)$ \\
UPDRS II [mean \pm SD (range)] & $14.08 \pm 7.14(2-31)$ \\
UPDRS III [mean \pm SD (range)] & $34.56 \pm 18.43(8-69)$ \\
HY [mean \pm SD (range)] & $2.44 \pm 0.69(1-4)$ \\
S\&E in \% [mean \pm SD (range)] & $77.95 \pm 11.96(50-100)$ \\
Medications in use [frequency (\%)] & $37(92.50)$ \\
Levodopa & $20(50.00)$ \\
Pramipexole & $7(17.50)$ \\
Entacapone & $11(27.50)$ \\
Amantadine & \\
\hline
\end{tabular}

HY: Hoehn and Yahr staging scale; PD: Parkinson's disease; SD: standard deviation; S \& E: Schwab and England activities of daily living scale; UPDRS: Unified Parkinson's Disease Rating Scale.

$\rho=-0.534, P=0.006$, resp.). Here again, educational level correlated positively with MMSE and FAB scores $(\rho=0.790$, $P<0.001$ and $\rho=0.771, P=0.001$, resp.).

3.2. Plasma Levels of Chemokines. As shown in Figure 1, there was no significant difference between PD patients and controls regarding plasma levels of the evaluated chemokines CCL11/eotaxin $(t=-0.967 ; P=0.34$, Student's $t$ test $)$, CCL24/ eotaxin-2 $(Z=-1,300 ; P=0.19$, Mann-Whitney test $)$, CXCL10/IP-10 ( $Z=-0.035 ; P=0.97$, Mann-Whitney test $)$, and CCL2/MCP-1 $(Z=-0.148 ; P=0.88$, Mann-Whitney test).

Circulating CXCL10/IP-10 levels were associated with PD progression since CXCL10/IP-10 plasma concentration correlated positively with HY staging scale $(\rho=0.366, P=0.026)$. Moreover, among PD patients increased CXCL10/IP-10 levels were associated with worse performance in cognitive tests; that is, CXCL10/IP-10 levels correlated negatively with MMSE $(\rho=-0.395, P=0.016)$ and FAB $(\rho=-0.458, P=0.004)$ scores (Figure 2). More specifically, increased CXCL10/IP-10 levels were associated with decreased mental flexibility $(\rho=$ $-0.439, P=0.007)$ and inhibitory control $(\rho=-0.365$, $P=0.026)$. It is worth mentioning that the correlation between CXCL10/IP-10 levels and total FAB score remained significant even after Bonferroni's correction. We found no correlation between plasma levels of chemokines and BDI or S\&E scores. In addition, we found no association between chemokines levels and clinical or demographic data in control subjects.

\section{Discussion}

This report demonstrated that PD patients and control individuals present comparable plasma concentrations of the chemokines CCL2/MCP-1, CCL11/eotaxin, CCL24/eotaxin2, and CXCL10/IP-10. Interestingly, increased CXCL10/IP10 levels were associated with PD progression and worse performance in cognitive tests.
Only few studies have evaluated circulating levels of chemokines in PD and the results are inconclusive. Our work group has already measured serum levels of chemokines in an independent cohort of PD patients, finding no significant difference between $\mathrm{PD}$ patients and age- and gender-matched controls [31], corroborating the current data. Rentzos and colleagues [14] evaluated serum levels of CCL5/RANTES in $41 \mathrm{PD}$ patients and 19 controls. PD patients presented higher levels of CCL5/RANTES in comparison with controls, and circulating levels of CCL5/RANTES positively correlated with UPDRS III scores. Remarkably, untreated patients $(n=$ 20) showed higher levels of CCL5/RANTES than control and treated PD groups [14], indicating that PD-related drugs may interfere with chemokines levels.

Another study has demonstrated that basal and lipopolysaccharide- (LPS-) induced levels of the chemokines CCL2/MCP-1, CCL3/MIP-1 $\alpha$, and CXCL8/IL- 8 and basal levels of CCL5/RANTES were significantly higher in PD patients than in controls. In addition, chemokines levels were associated with PD severity, since they correlated positively with UPDRS III scores and HY stages [15]. It is worth mentioning that, although all patients were taking antiparkinsonian drugs, as well as in our study, they used a different strategy to assess peripheral levels of chemokines; that is, they measured the levels of chemokines produced by peripheral blood mononuclear cells instead of serum/plasma quantifications [15].

To the best of our knowledge, the current study is the first to describe the association between peripheral chemokines levels and cognitive performance in PD. A few studies have assessed the association between chemokines and nonmotor symptoms in PD. One recent study evaluated the association between nonmotor symptoms and chemokines assessed in the cerebrospinal fluid (CSF) of PD patients [32]. With this purpose, CCL11/eotaxin, CXCL-10/IP-10, CCL2/MCP1 , and CCL4/MIP- $1 \beta$ levels were measured in the CSF of 87 PD patients (16 with dementia diagnosis) and 33 controls. Corroborating our results, there was no difference between groups for any of the measured CSF chemokines. 


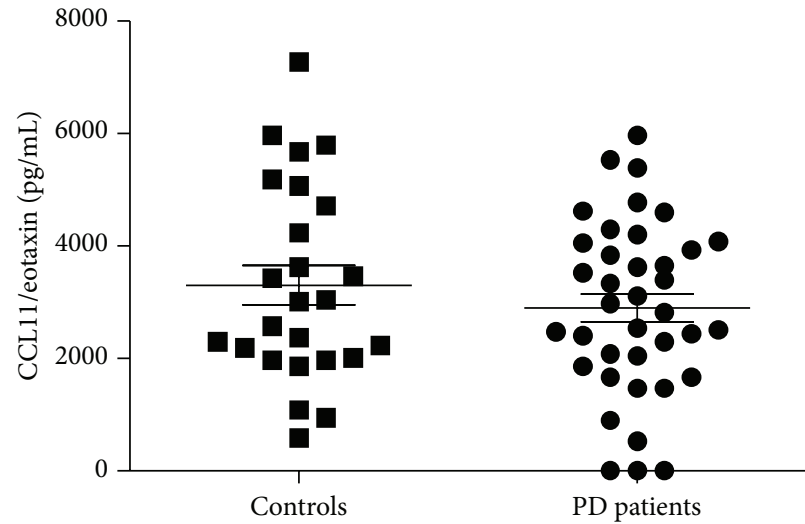

(a)

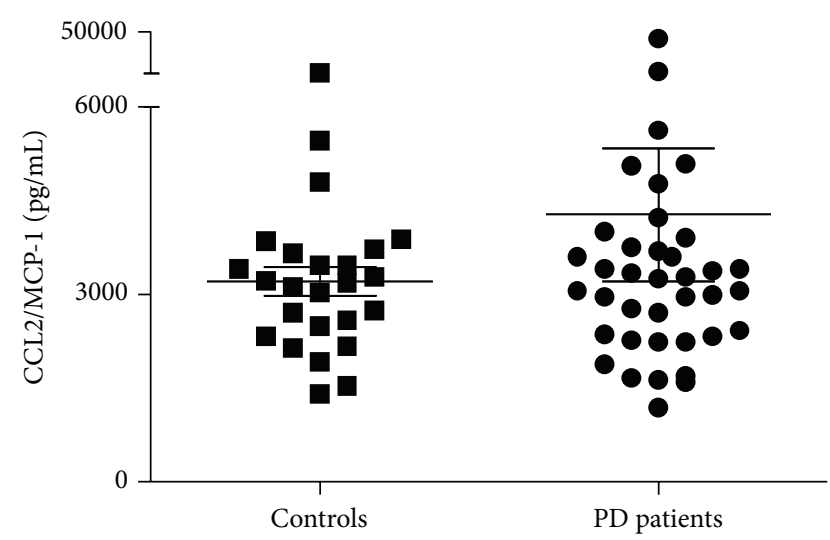

(c)

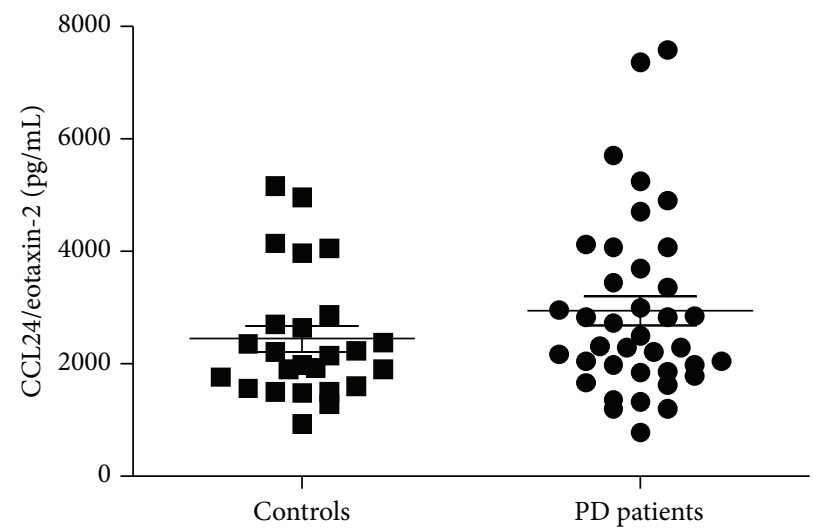

(b)

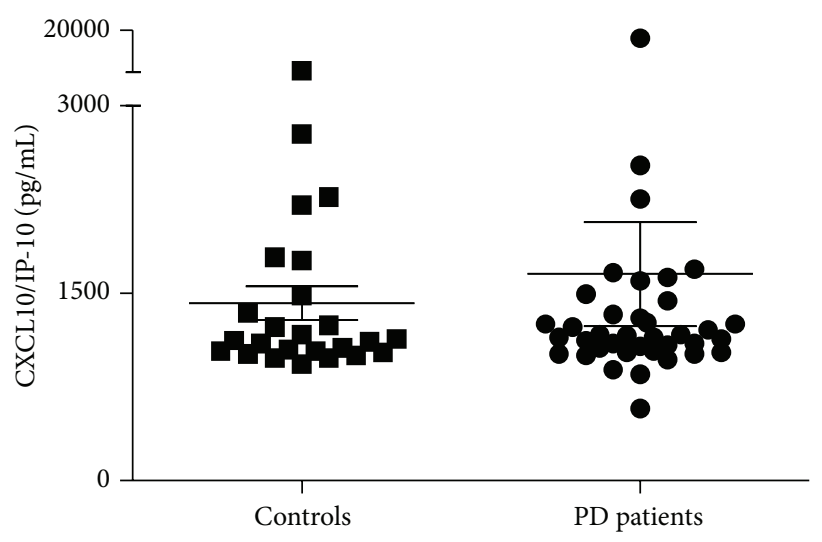

(d)

Figure 1: Control individuals and Parkinson's disease (PD) patients did not differ regarding plasma levels of the evaluated chemokines CCL11/eotaxin (a), CCL24/eotaxin-2 (b), CCL2/MCP-1 (c), and CXCL10/IP-10 (d). The figure shows mean and standard deviation of the mean (SEM). IP-10: interferon gamma-induced protein-10; MCP-1: monocyte chemotactic protein 1; PD: Parkinson's disease.

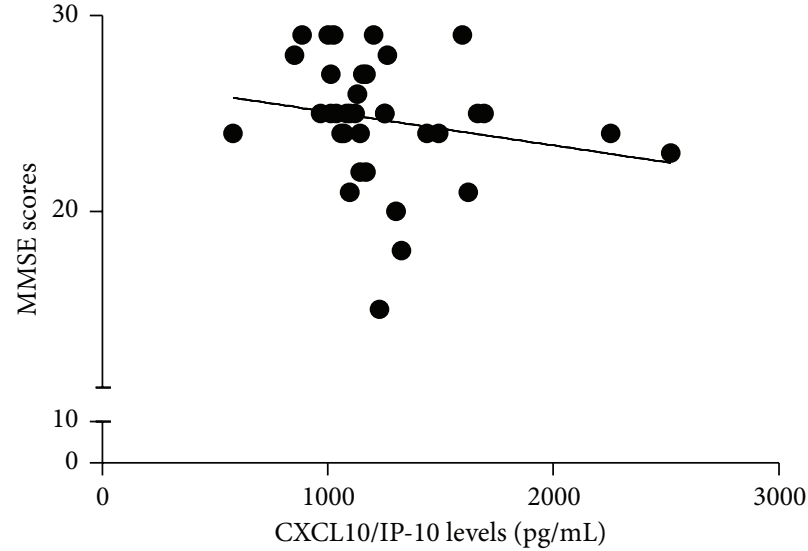

(a)

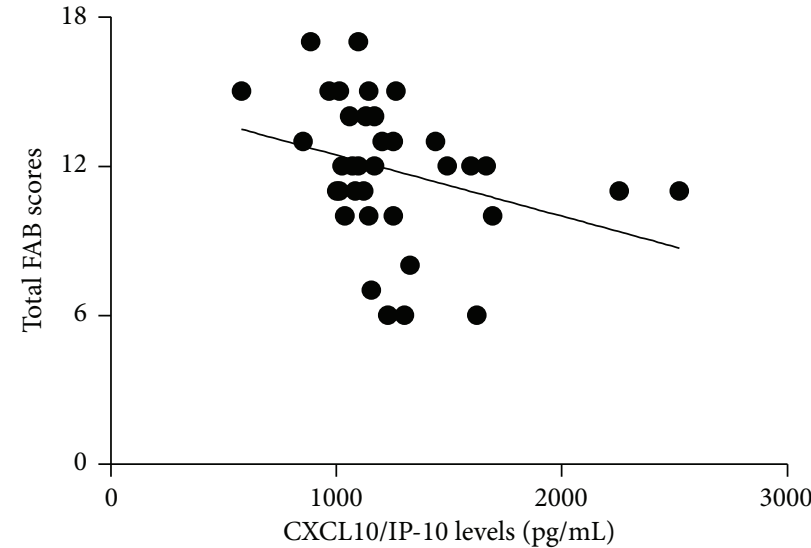

(b)

Figure 2: Among Parkinson's disease (PD) patients, plasma levels of CXCL10/IP-10 were inversely associated with MMSE (a; $\rho=-0.395, P=$ $0.016)$ and total FAB scores ( $b ; \rho=-0.458, P=0.004)$. IP-10: interferon gamma-induced protein-10; MMSE: Mini-Mental State Examination; FAB: Frontal Assessment Battery. 
CCL2/MCP-1 levels correlated with symptoms of depression, as assessed by the Hospital Anxiety and Depression Scale, and UPDRS motor score. FACIT-fatigue score correlated negatively with CXCL10/IP-10 and CCL2/MCP-1 levels; that is, the higher the levels of chemokines the more severe the fatigue symptoms. They did not find any significant correlation between cognitive performance as assessed by the MMSE and the evaluated chemokines [32]. Another study measured CX3CL1/fractalkine levels in the CSF of 126 $\mathrm{PD}$ patients in comparison with multiple systems atrophy patients $(n=32)$, AD patients $(n=50)$, and age-matched controls $(n=137)$ [33]. Again, there was no significant difference between groups regarding chemokine CSF levels. CSF fractalkine/amyloid- $\beta$ ratio increased significantly with increasing UPDRS scores, HY stage, and being a putative biomarker for PD severity. In an independent cohort comprising $39 \mathrm{PD}$ patients, CX3CL1/fractalkine alone correlated with the clinical progression of PD [33]. Interestingly, an experimental study showed that this chemokine is neuroprotective in MPTP-induced PD mice [34].

We found that CXCL10/IP-10 levels were associated with PD progression. The chemokine CXCL10/IP-10 has already been associated with PD pathophysiology, since IL- $1 \beta$ induced CXCL10/IP-10 protein expression was potentiated by coexposure to $\alpha$-synuclein in human A172 astroglial cells [35]. The chemokine CXCL10/IP-10 has also been associated with cognitive status in $\mathrm{AD}$, the prototype cognitive disorder. CSF CXCL10/IP-10 concentration was significantly increased in patients with mild cognitive impairment and mild $\mathrm{AD}$ in comparison with controls, but not in patients with severe AD. There was a significant positive correlation between MMSE score and CSF CXCL10/IP-10 levels [36]. Nonetheless, the same authors showed that serum levels of CXCL10/IP10 were not increased in mild cognitive impairment and $\mathrm{AD}$ regardless of the stage of the disease. In addition, no correlation between serum CXCL10/IP-10 levels and MMSE scores or the duration of the disease was found [37]. These observations suggest that CSF CXCL10/IP-10 levels increase may be restricted to an early stage of the disease, when proinflammatory events are thought to play a more relevant role in its pathogenesis [37]. Since our sample is composed by patients with moderate to advanced PD (mean age = 68 years; mean disease duration $=5.5$ years; mean total UPDRS score $=51.8$; mean motor UPDRS score $=34.6$ ), it is not possible to test this hypothesis, which deserves further investigation.

Limitations of our study include sample size and the fact that all patients were under anti-parkinsonian treatment that may influence the results. However, statistical analysis showed that the use of antiparkinsonian or other drugs did not influence chemokines levels in the present study. We also have to take into account that only peripheral blood levels of chemokines were evaluated, and they did not necessarily reflect what it is going on in the CNS. The parallel assessment of chemokines in the CSF would be interesting. By contrast, the strict exclusion criteria, the selection of controls with comparable age, gender, and BMI, and the analysis of clinical and inflammatory parameters together can be regarded as strengths of the study.
Although there was no significant difference between PD patients and control individuals regarding plasma levels of chemokines, our results showed that CXCL10/IP-10 levels may be associated with cognitive status in PD. Additional studies are needed in order to elucidate chemokines role in $\mathrm{PD}$, mainly regarding cognitive impairment.

\section{Conflict of Interests}

The authors declare that there is no conflict of interests regarding the publication of this paper.

\section{Authors' Contribution}

Natália Pessoa Rocha, Antônio Lúcio Teixeira, and Helton José Reis worked on the conception and organization of the research project. Natália Pessoa Rocha, Paula Luciana Scalzo, Izabela Guimarães Barbosa, Mariana Soares Souza, Isabela Boechat Morato, Érica Leandro Marciano Vieira, and Paulo Pereira Christo worked on the execution of the research project. Natália Pessoa Rocha and Antônio Lúcio Teixeira designed and executed the statistical analyses and wrote the first draft of the paper. Antônio Lúcio Teixeira and Helton José Reis reviewed the statistical analyses and the paper. Antônio Lúcio Teixeira and Helton José Reis contributed equally to the study.

\section{Acknowledgments}

The authors would like to acknowledge the participation of volunteers in this study and are indebted to their caregivers for their magnificent support. They also would like to thank the Members of LIIM for their critical comments and skilled technical assistance. This study was supported by FAPEMIG, CAPES, and CNPq, Brazil. Natália Pessoa Rocha is currently a CAPES scholarship recipient. Érica Leandro Marciano Vieira and Izabela Guimarães Barbosa are Postdoctoral Fellows of $\mathrm{CNPq}$ and CAPES, respectively. Isabela Boechat Morato is a CNPq undergraduate scholarship recipient. Antônio Lúcio Teixeira and Helton José Reis are CNPq fellowship recipients.

\section{References}

[1] A. Samii, J. G. Nutt, and B. R. Ransom, "Parkinson's disease," The Lancet, vol. 363, no. 9423, pp. 1783-1793, 2004.

[2] L. M. Bekris, I. F. Mata, and C. P. Zabetian, "The genetics of Parkinson disease," Journal of Geriatric Psychiatry and Neurology, vol. 23, no. 4, pp. 228-242, 2010.

[3] J. Parkinson, "An essay on the shaking palsy. 1817," The Journal of Neuropsychiatry and Clinical Neurosciences, vol. 14, no. 2, pp. 223-236, 2002.

[4] P. L. McGeer, S. Itagaki, B. E. Boyes, and E. G. McGeer, "Reactive microglia are positive for HLA-DR in the substantia nigra of Parkinson's and Alzheimer's disease brains," Neurology, vol. 38, no. 8, pp. 1285-1291, 1988.

[5] L. M. Collins, A. Toulouse, T. J. Connor, and Y. M. Nolan, "Contributions of central and systemic inflammation to the pathophysiology of Parkinson's disease," Neuropharmacology, vol. 62, no. 7, pp. 2154-2168, 2012. 
[6] D. Lindqvist, E. Kaufman, L. Brundin, S. Hall, Y. Surova, and O. Hansson, "Non-motor symptoms in patients with Parkinson's disease-correlations with inflammatory cytokines in serum," PLoS ONE, vol. 7, no. 10, Article ID e47387, 2012.

[7] Z. Katsarou, S. Bostantjopoulou, O. Hatzizisi, E. Giza, A. SolerCardona, and G. Kyriazis, "Immune factors or depression? Fatigue correlates in Parkinson's disease," Revista de Neurologia, vol. 45, no. 12, pp. 725-728, 2007.

[8] M. Menza, R. D. Dobkin, H. Marin et al., "The role of inflammatory cytokines in cognition and other non-motor symptoms of Parkinson's disease," Psychosomatics, vol. 51, no. 6, pp. 474-479, 2010.

[9] A. S. Chen-Plotkin, W. T. Hu, A. Siderowf et al., "Plasma epidermal growth factor levels predict cognitive decline in Parkinson disease," Annals of Neurology, vol. 69, no. 4, pp. 655-663, 2011.

[10] A. Réaux-Le Goazigo, J. van Steenwinckel, W. Rostène, and S. Mélik Parsadaniantz, "Current status of chemokines in the adult CNS," Progress in Neurobiology, vol. 104, pp. 67-92, 2013.

[11] A. Guyon, D. Skrzydelski, I. De Giry et al., "Long term exposure to the chemokine CCL2 activates the nigrostriatal dopamine system: a novel mechanism for the control of dopamine release," Neuroscience, vol. 162, no. 4, pp. 1072-1080, 2009.

[12] M. Shimoji, F. Pagan, E. B. Healton, and I. Mocchetti, "CXCR4 and CXCL12 expression is increased in the nigro-striatal system of Parkinson's disease," Neurotoxicity Research, vol. 16, no. 3, pp. 318-328, 2009.

[13] O. A. Ross, C. O’Neill, I. M. Rea et al., "Functional promoter region polymorphism of the proinflammatory chemokine IL8 gene associates with Parkinson's disease in the Irish," Human Immunology, vol. 65, no. 4, pp. 340-346, 2004.

[14] M. Rentzos, C. Nikolaou, E. Andreadou et al., "Circulating interleukin-15 and RANTES chemokine in Parkinson's disease," Acta Neurologica Scandinavica, vol. 116, no. 6, pp. 374-379, 2007.

[15] M. Reale, C. Iarlori, A. Thomas et al., "Peripheral cytokines profile in Parkinson's disease," Brain, Behavior, and Immunity, vol. 23, no. 1, pp. 55-63, 2009.

[16] R. Grassi-Oliveira, E. Brieztke, A. Teixeira et al., "Peripheral chemokine levels in women with recurrent major depression with suicidal ideation," Revista Brasileira de Psiquiatria, vol. 34, no. 1, pp. 71-75, 2012.

[17] I. G. Barbosa, N. P. Rocha, M. E. Bauer et al., "Chemokines in bipolar disorder: trait or state?" European Archives of Psychiatry and Clinical Neuroscience, vol. 263, no. 2, pp. 159-165, 2013.

[18] E. Asevedo, A. Gadelha, C. Noto et al., "Impact of peripheral levels of chemokines, BDNF and oxidative markers on cognition in individuals with schizophrenia," Journal of Psychiatric Research, vol. 47, no. 10, pp. 1376-1382, 2013.

[19] L. F. Fontenelle, I. G. Barbosa, J. V. Luna, L. P. de Sousa, M. N. S. Abreu, and A. L. Teixeira, "A cytokine study of adult patients with obsessive-compulsive disorder," Comprehensive Psychiatry, vol. 53, no. 6, pp. 797-804, 2012.

[20] H. D. Soares, W. Z. Potter, E. Pickering et al., "Plasma biomarkers associated with the apolipoprotein E genotype and alzheimer disease," Archives of Neurology, vol. 69, no. 10, pp. 1310-1317, 2012.

[21] D. Aarsland, S. Påhlhagen, C. G. Ballard, U. Ehrt, and P. Svenningsson, "Depression in Parkinson disease-epidemiology, mechanisms and management," Nature Reviews Neurology, vol. 8, no. 1, pp. 35-47, 2012.

[22] P. Bugalho and J. Vale, "Brief cognitive assessment in the early stages of parkinson disease," Cognitive and Behavioral Neurology, vol. 24, no. 4, pp. 169-173, 2011.
[23] A. Kummer and A. L. Teixeira, "Neuropsychiatry of Parkinson's disease," Arquivos de Neuro-Psiquiatria, vol. 67, no. 3, pp. 930939, 2009.

[24] M. J. Stuart and B. T. Baune, "Chemokines and chemokine receptors in mood disorders, schizophrenia, and cognitive impairment: a systematic review of biomarker studies," Neuroscience \& Biobehavioral Reviews, vol. 42, pp. 93-115, 2014.

[25] A. J. Hughes, S. E. Daniel, L. Kilford, and A. J. Lees, "Accuracy of clinical diagnosis of idiopathic Parkinson's disease: a clinicopathological study of 100 cases," Journal of Neurology, Neurosurgery \& Psychiatry, vol. 55, no. 3, pp. 181-184, 1992.

[26] S. Fahn and R. Elton, "Unified Parkinson's disease rating scale," in Recent Developments in Parkinson's Disease, S. Fahn, C. D. Marsden, D. B. Caine, and M. Goldstein, Eds., vol. 2, pp. 153163, 293-304, 1987.

[27] M. M. Hoehn and M. D. Yahr, "Parkinsonism: onset, progression and mortality.", Neurology, vol. 17, no. 5, pp. 427-442, 1967.

[28] M. F. Folstein, S. E. Folstein, and P. R. Mchugh, ““"Mini-mental state". A practical method for grading the cognitive state of patients for the clinician," Journal of Psychiatric Research, vol. 12, pp. 189-198, 1975.

[29] B. Dubois, A. Slachevsky, I. Litvan, and B. Pillon, “The FAB: a frontal assessment battery at bedside," Neurology, vol. 55, no. 11, pp. 1621-1626, 2000.

[30] A. T. Beck, C. H. Ward, M. Mendelson, J. Mock, and J. Erbaugh, "An inventory for measuring depression," Archives of General Psychiatry, vol. 4, pp. 561-571, 1961.

[31] P. Scalzo, A. S. de Miranda, D. C. Guerra Amaral, M. de Carvalho Vilela, F. Cardoso, and A. L. Teixeira, "Serum levels of chemokines in parkinson's disease," Neuroimmunomodulation, vol. 18, no. 4, pp. 240-244, 2011.

[32] D. Lindqvist, S. Hall, Y. Surova et al., "Cerebrospinal fluid inflammatory markers in Parkinson's disease-associations with depression, fatigue, and cognitive impairment," Brain, Behavior, and Immunity, vol. 33, pp. 183-189, 2013.

[33] M. Shi, J. Bradner, A. M. Hancock et al., "Cerebrospinal fluid biomarkers for Parkinson disease diagnosis and progression," Annals of Neurology, vol. 69, no. 3, pp. 570-580, 2011.

[34] J. M. Morganti, K. R. Nash, B. A. Grimmig et al., "The soluble isoform of CX3CL1 is necessary for neuroprotection in a mouse model of Parkinson's disease," The Journal of Neuroscience, vol. 32, no. 42, pp. 14592-14601, 2012.

[35] N. S. Tousi, D. J. Buck, J. T. Curtis, and R. L. Davis, “ $\alpha$-Synuclein potentiates interleukin- $\beta$-induced CXCL10 expression in human A172 astrocytoma cells," Neuroscience Letters, vol. 507, no. 2, pp. 133-136, 2012.

[36] D. Galimberti, N. Schoonenboom, P. Scheltens et al., "Intrathecal chemokine synthesis in mild cognitive impairment and Alzheimer disease," Archives of Neurology, vol. 63, no. 4, pp. 538543, 2006.

[37] D. Galimberti, E. Venturelli, C. Fenoglio et al., "IP-10 serum levels are not increased in mild cognitive impairment and Alzheimer's disease," European Journal of Neurology, vol. 14, no. 4, pp. e3-e4, 2007. 


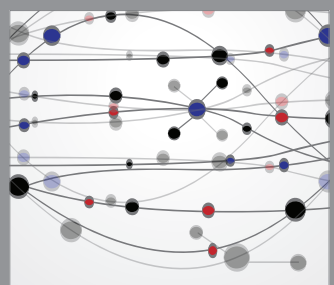

The Scientific World Journal
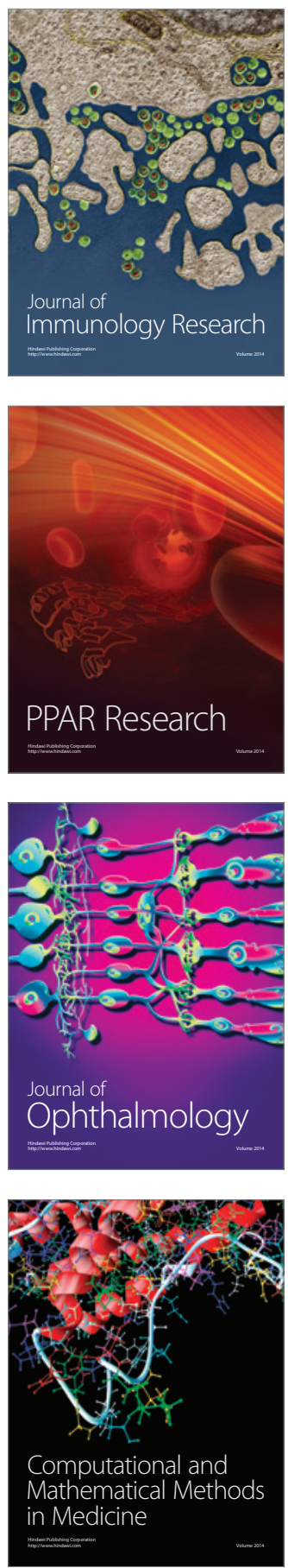

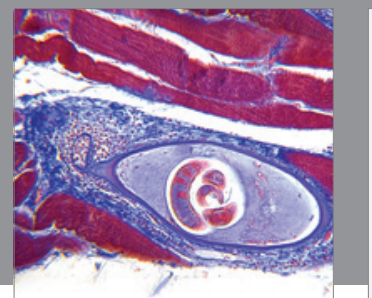

Gastroenterology

Research and Practice
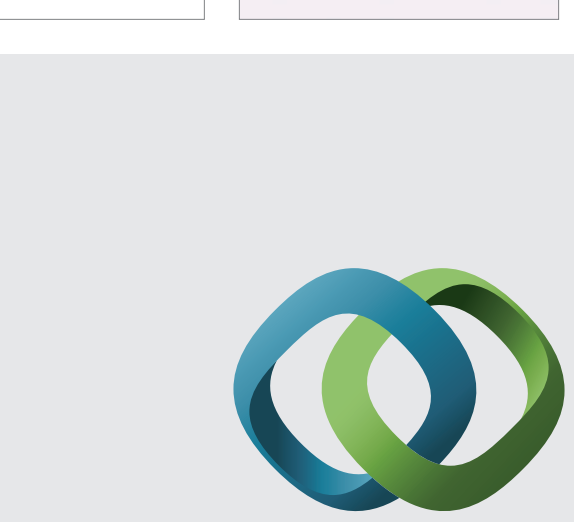

\section{Hindawi}

Submit your manuscripts at

http://www.hindawi.com
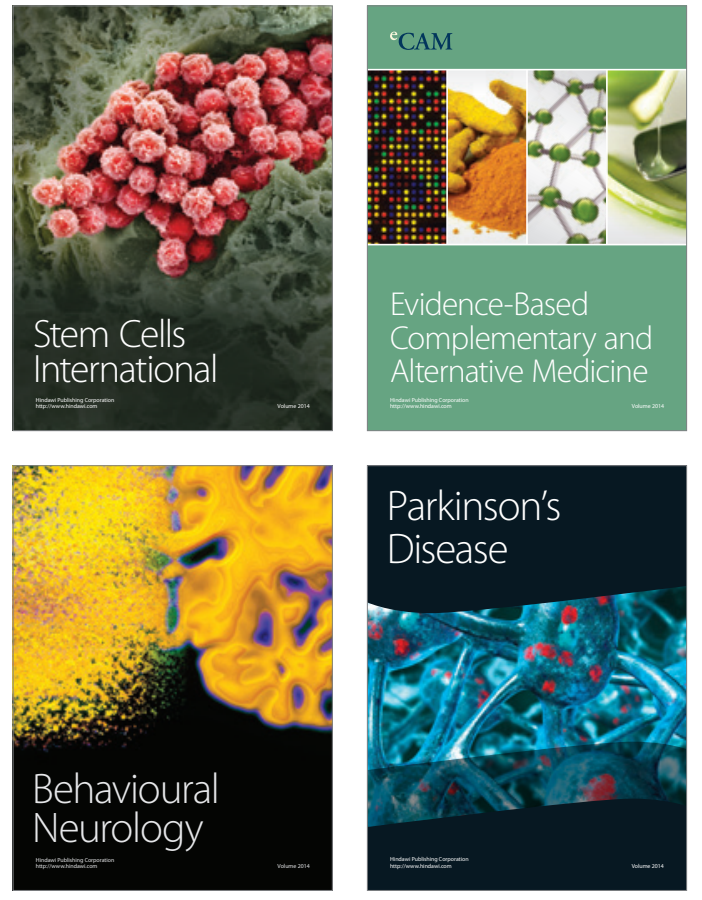
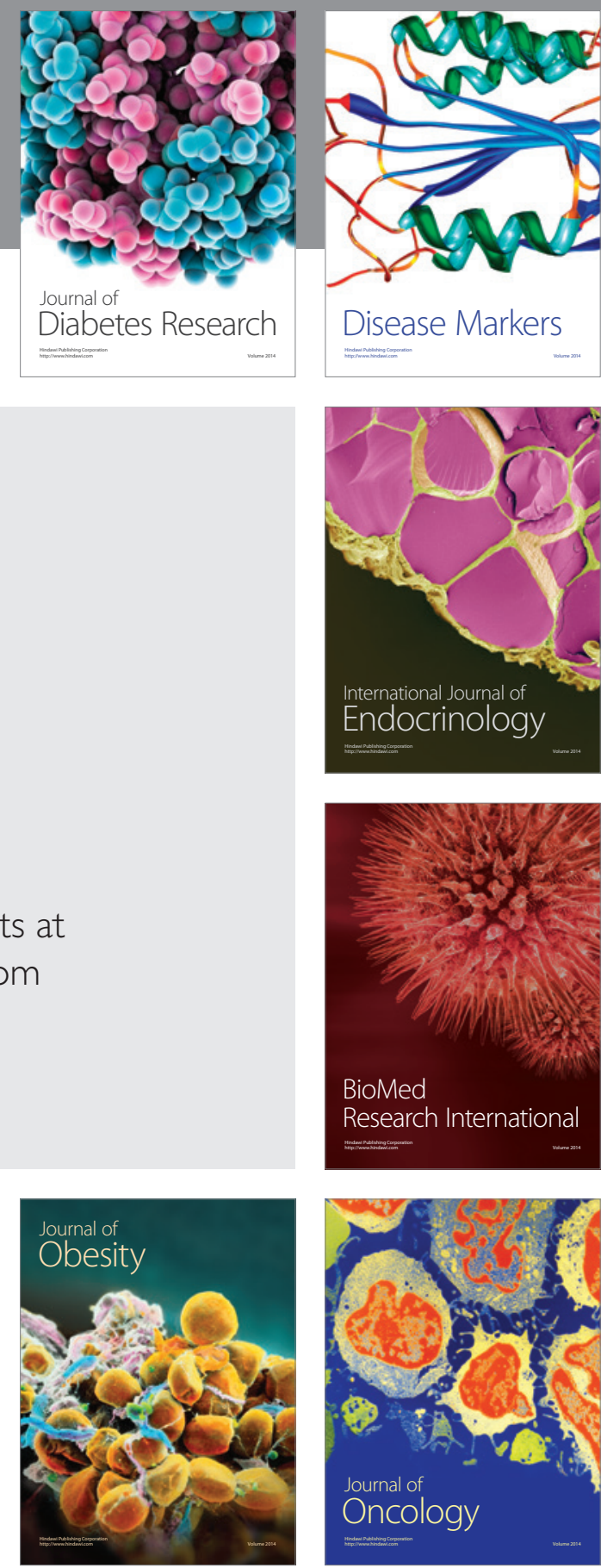

Disease Markers
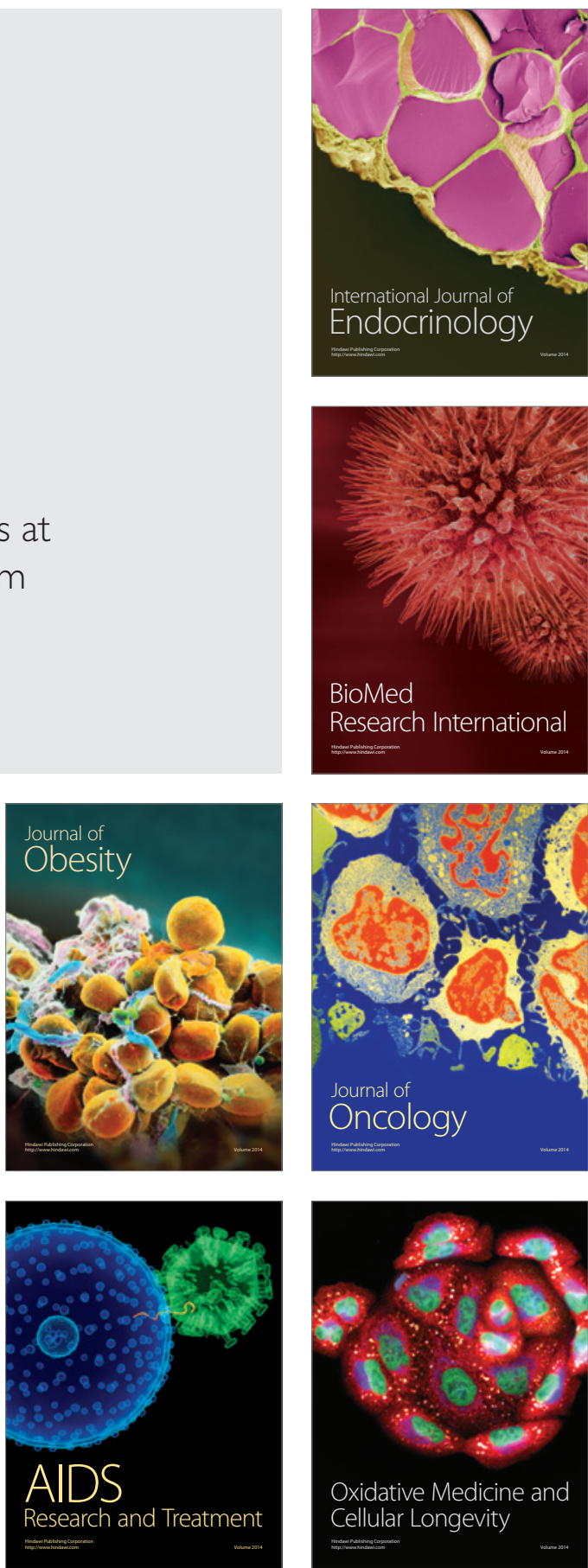